\section{Digitale Abformung}

\section{Neu und doch vertraut}

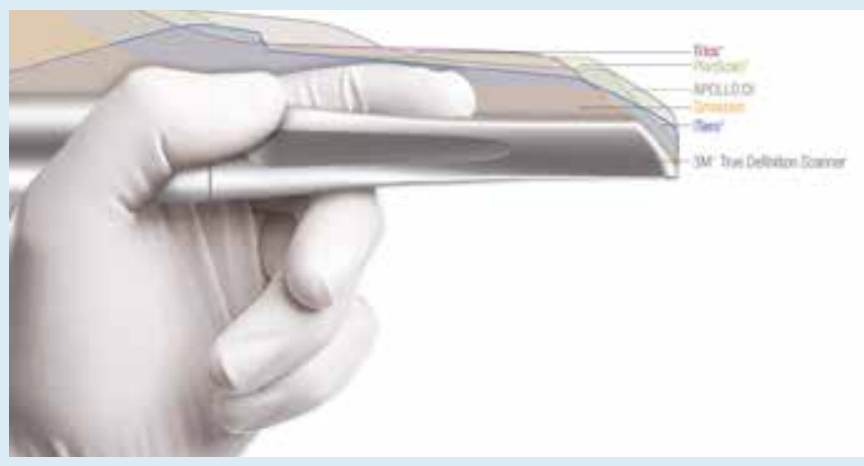

Vertrauens. Eine Vereinfachung des Scanprozesses wird u.a. durch das optimierte Design des Handstücks erzielt. Dieses ähnelt in seiner Form ei-

Um die modernen Anforderungen in der Zahnarztpraxis hinsichtlich Effizienz und Qualität zu erfüllen, sollte der Abformprozess einfach und schnell mit hoher Präzision durchführbar sein. Ermöglicht wird dies durch die Anwendung des neuen $3 \mathrm{M}$ True Definition-Scanners. Die erzeugten Daten können flexibel weiterverarbeitet werden - zum Beispiel im Labor des nem klassischen Winkelstück, liegt mit einem Gewicht von $230 \mathrm{~g}$ leicht in der Hand und lässt sich in einem Abstand von 0-18 mm einfach über die Zahnreihen führen. Aufgrund der abgerundeten, angewinkelten Spitze kann auch der hintere Molarenbereich sogar bei Patienten mit geringer Mundöffnung zuverlässig aufgenommen werden.
Dank dieses einfachen Handlings in Kombination mit Weiterentwicklungen der Software wird eine deutliche Verkürzung der Scanzeit gegenüber vorherigen Scanner-Generationen erreicht: Ein Ganzkieferscan lässt sich von einem geübten Anwender und nach sorgfältiger Trockenlegung in rund $60 \mathrm{~s}$ abschließen. Die erzielten Ergebnisse weisen Studien zufolge eine höhere (Wiederhol)-Genauigkeit auf als die mit anderen Intraoralscannern erzeugten Daten.

Da die Abformdaten im STL-Format verfügbar sind, können die Datensätze einfach an das Partnerlabor gesendet und dort z. B. für die virtuelle Konstruktion des Zahnersatzes verwendet werden. Zusätzlich sind über sogenannte „Trusted Connections“ validierte, nahtlose Workflows beispielsweise für verschiedene Implantatsysteme oder auch die Modellherstellung verfügbar.

Nach einer Pressemitteilung der

$3 \mathrm{M}$ Deutschland $\mathrm{GmbH}$, Seefeld 